\title{
Regeneración de Oreomunnea mexicana (Juglandaceae), una especie relicto, amenazada del bosque de niebla de Sierra Juárez, Oaxaca, México
}

\author{
Regeneration of Oreomunnea mexicana (Juglandaceae), a threatened \\ relict species of the Sierra Juarez cloud forest, Oaxaca, Mexico
}

\author{
Nelly Pacheco-Cruz', Ricardo Clark-Tapia², Jorge E. Campos', Montserrat Gorgonio-Ramírez², María Delfina Luna- \\ Krauletz ${ }^{2}$, Francisco Naranjo-Luna ${ }^{3}$, Adriana Corrales ${ }^{4}$ y Cecilia Alfonso-Corrado² ${ }^{*}$
}

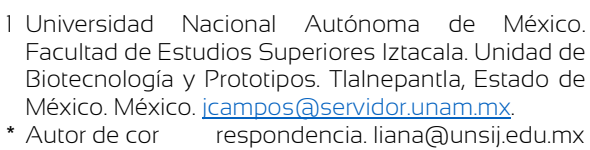

\author{
2 Universidad de la Sierra Juárez. Instituto de Estudios \\ Ambientales. Ixtlán de Juárez, Oaxaca, México. \\ rclark@unsij.edu.mx; montse_grmz@hotmail.com; \\ mkrauletz@unsij.edu.mx \\ 3 Instituto Tecnológico Superior de Jesús Carranza. \\ Veracruz, México. francobio@gmail.com.mx \\ 4 Universidad del Rosario. Bogotá, Colombia. \\ adricorrales33@gmail.com***
}

\begin{abstract}
RESUMEN
Oreomunnea mexicana (Stand.) J.F. Leroy es una especie relicto, amenazada, y clave para el funcionamiento del ecosistema de niebla de la Sierra Juárez, Oaxaca, el más extenso y conservado de México. En este estudio se realizó un diagnóstico en dos etapas críticas del proceso de regeneración poblacional de O. mexicana. Para ello, se analizó: 1) la producción, dispersión, viabilidad y germinación de semillas, 2) el efecto de la depredación en el crecimiento y supervivencia de las plántulas, y 3) el efecto de variables ambientales (suelo, estructura del dosel y radiación solar) en la regeneración de la especie. El diagnóstico se realizó de 2014 a 2015 en el bosque de niebla de Santiago Comaltepec, Oaxaca, México, dentro del cual se seleccionaron dos localidades: San Bernardo y El Orquidiario. Se encontraron diferencias en la producción de semillas entre años y entre localidades, además de baja viabilidad y un alto porcentaje de parasitismo en las semillas. El establecimiento y crecimiento de las plántulas están sujetos a encontrar microhábitats adecuados, dado que las plántulas tienen requerimientos específicos de iluminación y de minerales esenciales en el suelo. La alta especificidad edáfica que presenta la especie puede ser factor de su distribución fragmentada y discontinua en la Sierra Juárez. Se recomienda continuar con los estudios de regeneración poblacional para aumentar el conocimiento de la especie y con ello lograr su conservación.
\end{abstract}

PALABRAS CLAVE: conservación, crecimiento, establecimiento, iluminación, suelo, supervivencia.

\begin{abstract}
Oreomunnea mexicana (Stand.) J.F. Leroy is a relict species, threatened and a key species for cloud ecosystem functionof the Sierra Juárez, Oaxaca, the most extensive and best conserved in Mexico. This study includes a diagnostic on two critical stages of the process of population regeneration of $O$. mexicana. To this end, it analyzed the: 1) production, dispersion, viability and germination of seeds, 2) effect of predation on the growth and survival of the seedlings, and 3) effect of environmental variables (soil, canopy structure and solar radiation) on the regeneration of the species. The study was made from 2014 to 2015 in the cloud forest of Santiago Comaltepec, Oaxaca, Mexico, in two locations (San Bernardo and El Orquidiario). Differences in seed production were found between years and between locations, as well as low seed viability and a high percentage of seed parasitism. The establishment and growth of the seedlings are subject to find suitable microhabitats, since seedlings have specific illumination and soil requirements. The high edaphic specificity faced by the species could be a key factor for its fragmented and discontinuous distribution in Sierra Juárez. Further population regeneration studies are recommended to increase knowledge of the species and thereby achieve its conservation.
\end{abstract}

KEYWORDS: conservation, growth, establishment, illumination, soil, survival. 


\section{INTRODUCCIÓN}

La regeneración poblacional de una planta consiste en una serie de procesos demográficos cíclicos que van desde la producción, dispersión y germinación de semillas hasta el establecimiento y crecimiento de nuevos reclutas de una población que es afectada por procesos bióticos y abióticos (Harper, 1977; Pérez-López, López-Barrera, García-Oliva, Cuevas-Reyes y González-Rodríguez, 2013). Un fracaso o afectación en alguna de las etapas demográficas de una especie en cuestión disminuirá su regeneración natural y puede conducirla a la extinción (Parra-Aldana, DíezGómez y Moreno-Hurtado, 2011). Por ello, su conocimiento a corto y largo plazo es crucial en el diseño de estrategias de conservación de plantas, en particular de especies raras, endémicas y relictos (Primarck, 2012; Pacheco-Cruz et al., 2016).

En el ciclo de la regeneración poblacional, los procesos que ocurren durante los estadios de semillas y de plántulas tienen un gran impacto en el establecimiento de una población (Parra-Aldana et al., 2011; Alfonso-Corrado et al., 2014). Por ejemplo, el establecimiento y crecimiento de nuevos individuos depende de factores bióticos como: 1) la producción de semillas (Alfonso-Corrado et al., 2014), 2) la distancia de dispersión de la semillas, 3) depredación de plántulas (Pérez-López et al., 2013), 4) las asociaciones micorrízicas (Corrales, Arnold, Ferrer, Turner y Dalling, 2016a), 5) la perturbación antrópica (Alfonso-Corrado et al., 2017); mientras que factores abióticos como son condiciones edáficas, radiación solar y microclima determinan la continuidad de una población en espacio y tiempo (Pérez-López et al., 2013; Alfonso-Corrado et al., 2014, Avendaño-Yáñez, Sánchez-Velázquez, Meave y Pineda-López, 2016).

Oreomunnea mexicana (Stand.) J.F. Leroy es una especie relicto del mioceno tardío y plioceno temprano (PalacioChávez y Rzedowski, 1993) que pertenece a la familia de las juglandáceas americanas y es la única especie representante del género en México (Russell y Cohn, 2012). Se distribuye de forma discontinua en los bosques de niebla localizados en los estados de Oaxaca, Chiapas y Veracruz (Palacio-
Chávez y Rzedowski, 1993). En Oaxaca se encuentra en el bosque de niebla de la Sierra Juárez formando fragmentos de poblaciones monodominantes con árboles que alcanzan hasta $40 \mathrm{~m}$ de altura, por lo que se considera una especie clave en el mantenimiento de la biodiversidad asociada (Alfonso-Corrado et al., 2017). Diversos estudios, entre ellos genéticos (Pascual-Mendoza, 2014), sobre su distribución actual, potencial y cambios ante escenarios climáticos (Alfonso-Corrado et al., 2017) y de demografía (Pacheco-Cruz et al., 2018) indican que la especie se encuentra amenazada y es vulnerable a cambios extremos en el ambiente. Por ejemplo, en marzo de 2016, un evento de fuertes vientos superiores a los $100 \mathrm{~km} \mathrm{~h}^{-1}$ provocó el derribo de árboles y la desaparición de 60\% a 70\% de la población total en tres localidades, lo que afectó su dinámica poblacional (Pacheco-Cruz et al., 2018).

En México, los estudios de regeneración en Oreomunnea mexicana se han focalizado en el estado de Veracruz donde se ha analizado la germinación de semillas (Atondo-Bueno, López-Barrera, Bonilla-Moheno, Williams-Linera y Ramírez-Marcial, 2016), así como el crecimiento y la supervivencia de plántulas (Avendaño-Yáñez et al., 2016). Atondo-Bueno, et al. (2016) registraron que la especie presenta una germinación menor a $50 \%$, ya sea en campo $(37 \%)$ o en laboratorio (47\%), señalan además que la emergencia de plántulas es afectada por características del suelo como la humedad e incremento de la cobertura arbórea. Por otra parte, Avendaño-Yáñez et al. (2016) encontraron que la supervivencia de plántulas es favorecida por la cobertura de árboles, no obstante, su crecimiento es mayor en espacios abiertos, por lo que sugieren una tolerancia de las plántulas a la radiación solar. Estos autores señalan que la principal causa de mortalidad en plántulas de O. mexicana es la herbivoría en el bosque de niebla.

En Oaxaca no existen estudios de regeneración de $O$. mexicana, por lo que un diagnóstico de la regeneración de esta especie es un proceso importante para garantizar programas de reforestación y conservación (AlfonsoCorrado et al., 2014), particularmente, considerando que el bosque de niebla de la Sierra Juárez, Oaxaca ha sido catalogado como el más extenso y conservado del país 
(Anta-Fonseca et al., 2010, Ponce-Reyes et al., 2012), que trasciende por su alta capacidad de almacenar carbono (Álvarez-Arteaga, García-Calderón, Krasilnikov y GarcíaOliva, 2013) y que es la fuente principal de agua de la cuenca del Río Papaloapan, la segunda más grande de México (Clark-Tapia, Suárez-Mota, Alfonso-Corrado, AguirreHidalgo y Campos-Contreras, 2016). Por todo lo anterior, resalta su importancia en servicios ambientales a escala nacional. En este contexto, Atondo-Bueno et al. (2016) señalan la necesidad de contar con información sobre factores ambientales que afectan la germinación de semillas y desarrollo de las plántulas en especies amenazadas o con distribución restringida y discontinua, como O. mexicana.

\section{OBJETIVOS}

Realizar un diagnóstico en dos etapas críticas del proceso de regeneración poblacional de O. mexicana: crecimiento y supervivencia de plántulas. Para ello, se analizaron: 1) producción, dispersión, viabilidad y germinación de semillas, 2) crecimiento, supervivencia y depredación de plántulas y 3) efecto de variables ambientales (suelo, estructura del dosel y radiación solar) en las dos etapas críticas mencionadas.

\section{MATERIALES Y MÉTODOS}

\section{Área de estudio}

El estudio se realizó en el bosque nuboso del municipio de Santiago Comaltepec, ubicado en la Sierra Juárez, Oaxaca, en el extremo sur de México, de donde se seleccionaron dos poblaciones: El Orquidiario (17 $37^{\circ} 46.06^{\prime \prime} \mathrm{N}$ y $96^{\circ} 21^{\prime} 2$ 6.28" O) y San Bernardo (17 36' 47.45" N y 96² 22' 37.72"

O) (Fig. 1). En estas localidades, la estructura del dosel muestra una monodominancia de O. mexicana, en asociación con otras especies arbóreas como Pinus chiapensis (Martínez) Andersen, Ocotea effusa (C.D.F. Meisner) W.B. Hemsley, Quercus laurina (Humb et Bonpl.), Sphaeropteris horrida (Liebm.) R.M. Tryon, Clethra integerrima (Turcz.) L.M. González, Ticodendron incognitum (Gómez-Laur. y L.D. Gómez) y Zinowiewia spp.
La especie tiene una distribución restringida a pequeños fragmentos menores de $1.0 \mathrm{~km}^{2}$, con características similares en cuanto a altitud (de $1704 \mathrm{~m}$ a $1750 \mathrm{~m} \mathrm{snm}$ ), orientación noroeste y pendiente entre $25 \%$ y 30\% (Alfonso-Corrado et al., 2017). El clima del área de estudio es semicálido húmedo con abundantes lluvias en verano; temperatura media anual de $18{ }^{\circ} \mathrm{C}$ y precipitación media anual de $1500 \mathrm{~mm}$ (Clark-Tapia et al., 2016). Sin embargo, una diferencia entre ambas localidades es su nivel de disturbio; El Orquidiario se encuentra ubicado a escasos $800 \mathrm{~m}$ de la comunidad, por lo cual es visitado frecuentemente y utilizado para consumo local de leña. En contraste, San Bernardo se encuentra a más de $3 \mathrm{~km} \mathrm{y}$ presenta mínima evidencia de disturbio humano.

\section{Descripción de la especie}

Es un árbol relicto de la era cenozoica de la familia Juglandaceae, que se distribuye de forma discontinua en México (Oaxaca, Chiapas y Veracruz), Guatemala, Nicaragua, Panamá, Costa Rica. Se encuentra entre los 1100 $\mathrm{m}$ y $2000 \mathrm{~m}$ snm. Alcanza una altura superior a los $30 \mathrm{~m}$ y se distingue por su corteza exfoliada. Tiene hojas perennes pinnadas desde cuatro hasta ocho foliolos y sus frutos son indehiscentes, bien protegidos y de solo una semilla recalcitrante (Stone, 1972; Russell y Cohn, 2012). La fructificación se ha registrado de octubre a enero (AtondoBueno et al., 2016); sin embargo, en la región de estudio ocurre de agosto a noviembre. La semilla se caracteriza por estar cubierta por una larga bráctea trilobada glabra, y por ser, además, especializados para anemocoria (Stone, 1972; Russell y Cohn, 2012). La germinación de las semillas es hipogea y se ve afectada por la infestación de escarabajos de la familia Curculionidae. La emergencia de plántulas se ha correlacionado positivamente con el contenido de humedad del suelo, pero negativamente con la cubierta vegetal, debido a que se desarrolla bien en microhábitats con intensidad de luz reducida (Stone, 1972; Atondo-Bueno et al., 2016). 


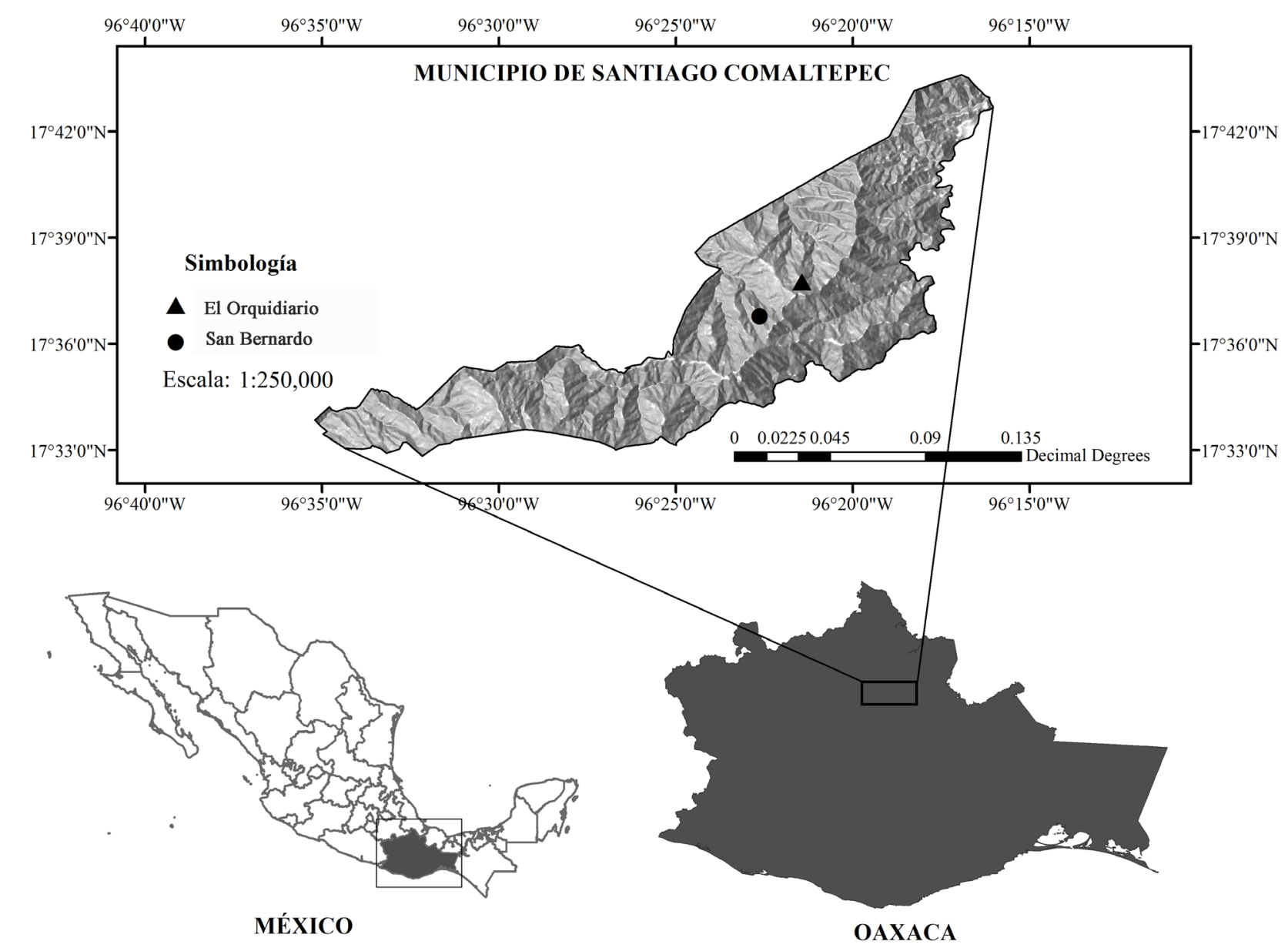

FIGURA 1. Ubicación de las localidades de estudio en el municipio de Santiago Comaltepec, Oaxaca, México.

\section{Producción de semillas}

La recolección y estimación de la producción de semillas para el estudio se realizó durante todo el mes de septiembre de los años 2014 y 2015 en las dos localidades de estudio, San Bernardo y El Orquidiario (Fig. 1). Dado que la dispersión de las semillas es por viento y la altura de los árboles es mayor a $30 \mathrm{~m}$, la recolección y producción de semillas se llevó a cabo con 20 trampas de un $1 \mathrm{~m}^{2}$ elaboradas con tela de tule distribuidas sistemáticamente cada cinco metros bajo el dosel del bosque de O. mexicana. De manera adicional, con la finalidad de determinar diferencias en el tamaño de las semillas entre años y localidades, se seleccionaron aleatoriamente 100 semillas de cada sitio por año para establecer una relación entre el peso y el tamaño (largo y ancho). Cada semilla fue pesada en una balanza de precisión AND (SHS) GF-300. Se realizó un análisis de varianza con prueba de contraste de Tukey para determinar diferencias significativas en la producción de semillas entre localidades y años, así como para determinar diferencias en el tamaño de semillas entre los años y las localidades de estudio.

\section{Viabilidad de las semillas (rayos $X$ )}

El diagnóstico de la viabilidad con rayos X solo se realizó en el año 2015, para ello se evaluó el número de semillas sanas, con daño estructural o malformadas y vanas (sin 
embrión o vacías). Del total de semillas recolectadas, se seleccionó una muestra aleatoria de 70 semillas, divididas en cinco repeticiones. Estas se colocaron en una cámara de irradiación no digital Faxitron X-Ray (Specimen Radiography System) modelo MX-20®, a una intensidad de irradiación de $18 \mathrm{kV}$ y el revelado de las placas se realizó con una impresora digital modelo 319 Micro-Max ${ }^{\circledR}$. Este análisis se realizó en el laboratorio de semillas del banco de germoplasma de la Secretaría de Desarrollo Agropecuario, Forestal, Pesca y Acuacultura del Gobierno del Estado de Oaxaca, México.

\section{Distancia de dispersión de semillas}

Debido a lo complejo que resulta estimar la dispersión de semillas por viento en campo (altura de los árboles, densidad del dosel, etc.), y dado que hubo mayor producción de semillas en ese año, esta se evalúo ex situ solamente para 2015. La evaluación se realizó utilizando una antena de radio comunicaciones de $30 \mathrm{~m}$ de altura. A pesar de que el método utilizado no es apegado a la realidad, puede proporcionar una idea de la distancia de dispersión y la factibilidad de evitar competencia intraespecífica o la habilidad de colonizar nuevas localidades en el mismo o diferente hábitat, aspectos importantes en la regeneración.

Para analizar la dispersión por viento de O. mexicana se seleccionaron al azar 100 semillas (50 de cada sitio de estudio). Esta selección se basó en elegir semillas sin evidencia de infestación y cuya bráctea trilobada no mostrara daño físico. Todas las semillas fueron marcadas con plumón indeleble, pesadas en una balanza de precisión AND (SHS) GF-300 y medidas en ancho y largo de la bráctea. Posteriormente, las semillas fueron lanzadas desde la torre en dirección del viento hacia el este a una velocidad de $2.6 \mathrm{~km} \mathrm{~h}^{-1}$ señalada por una estación meteorológica Davis Vantage Pro2, con la finalidad de conocer la distancia de dispersión, la cual fue registrada para cada semilla. Se aplicó una regresión lineal para determinar la relación entre el peso de las semillas y tamaño de bráctea en la distancia de dispersión usando el programa estadístico XLSTAT v. 1.0.1 (Addinsoft, 2014).
Crecimiento, supervivencia y depredación de plántulas de $\mathrm{O}$. mexicana

En noviembre de 2014, en las localidades de El Orquidiario y San Bernardo se estableció un área de muestreo al centro de la población de $20 \mathrm{~m} \times 20 \mathrm{~m}\left(400 \mathrm{~m}^{2}\right)$, subdividida en parcelas numeradas de $1 \mathrm{~m}^{2}$. En cada localidad se seleccionaron, con números aleatorios, 60 parcelas de $1 \mathrm{~m}^{2}$ dentro de las cuales se registraron la cantidad de semillas y plántulas germinadas con la finalidad de analizar el porcentaje de germinación in situ. Las plántulas registradas germinaron en el año 2014 y mostraron una altura promedio de $5.2 \mathrm{~cm} \pm 1.5 \mathrm{~cm}$ y foliolos desarrollados. De estas plántulas se seleccionaron 60, las cuales fueron numeradas y ubicadas geográficamente de manera individual con un geoposicionador GPS MobileMapper v. 10 (Ashtech) para seguir su crecimiento y supervivencia.

El crecimiento se evaluó de noviembre 2014 a junio 2015 con incremento mensual en la altura, diámetro del tallo y número de hojas. Para evaluar la supervivencia de las plántulas, se consideró que el tallo y las hojas de las plántulas no estuvieran secas o necrosadas y que las raíces estuvieran afianzadas al sustrato. La ausencia de una plántula numerada en evaluaciones posteriores fue considerada como muerta. Para analizar la depredación de estas 60 plántulas, 30 fueron excluidas de manera individual con una jaula de malla ciclónica de $60 \mathrm{~cm} \times 30 \mathrm{~cm}$, y 30 plántulas permanecieron sin ser excluidas (sin jaula).

Asimismo, para evaluar las diferencias en el crecimiento de las plántulas entre localidades (altura, diámetro del tallo y número de hojas), se realizó un análisis de varianza de una vía con pruebas de contraste de Tukey para cada variable utilizando el programa estadístico XLSTAT v.1.0.1 (Addinsoft, 2014). La supervivencia y depredación fueron analizadas con un análisis de varianza de una vía. Para ello, los datos absolutos de supervivencia se transformaron obteniendo la raíz cuadrada del arco seno, a fin de estabilizar la varianza antes de aplicar el análisis de varianza en el programa estadístico XLSTAT v.1.0.1 (Addinsoft, 2014). 


\section{Estructura del dosel y transmisión de la radiación en el sotobosque}

En el mes de septiembre de 2015, se realizó una estimación de variables de estructura del dosel y de luz para las localidades de El Orquidiario y San Bernardo mediante el software Gap Light Analyzer (GLA) v. 2.0 (Frazer, Canham y Lertzman, 1999), que simula el régimen diario de radiación solar y la estructura de la vegetación para el tiempo analizado. Esta estimación se realizó también para el análisis de depredación (con y sin exclusión), con la finalidad de evaluar si la exclusión de plántulas afectaba la cantidad de radiación y ello tenía un efecto en la supervivencia. Para el análisis se tomaron fotografías hemisféricas del dosel del bosque con una cámara réflex digital EOS 50D SLR y una lente semiesférica (ojo de pez) marca Canon EF f/4L USM, con apertura focal de $8 \mathrm{~mm}$ y apertura máxima de f 2.5. Este equipo se montó en un trípode, se aseguró la horizontalidad y posición de la lente a $1.5 \mathrm{~m}$ de altura, y se orientó para coincidir la parte superior de la fotografía con el Norte, de acuerdo con Quevedo, Schwarzkopf, García y Jerez (2016).

Se estimaron dos atributos de la estructura del dosel: a) el porcentaje de apertura del dosel $(\% \mathrm{AD})$, definido como el porcentaje de cielo abierto visto desde debajo de un dosel del bosque y b) el índice de área foliar (IAF) efectiva integrada sobre un ángulo de $0^{\circ}$ a $60^{\circ}$. Además, se calcularon tres atributos que describen la cantidad de radiación transmitida por el dosel del bosque: 1) porcentaje de radiación directa transmitida (\%RDT): proporción de luz directa transmitida a través del dosel con respecto a la radiación directa recibida por encima del dosel; 2) porcentaje de luz difusa transmitida (\%RFT): proporción de luz difusa transmitida a través del dosel con respecto a la radiación difusa recibida por encima del dosel; y 3) porcentaje de radiación total transmitida (\%RTT), es el promedio de \%RDT y \%RFT (Frazer et al., 1999). Se realizó un análisis de varianza de una vía con pruebas de contraste de Tukey para determinar diferencias entre localidades y entre condiciones excluidas y sin excluir en la incidencia lumínica usando el programa estadístico XLSTAT v.1.0.1 (Addinsoft, 2014).

\section{Análisis edáficos}

Con la finalidad de evaluar diversas variables físicas (humedad del suelo, densidad) y químicas (amonio $\left(\mathrm{NH}_{4}\right)$, nitrato $\left(\mathrm{NO}_{3}{ }^{-}\right)$, fósforo $(\mathrm{P})$, potasio $(\mathrm{K})$, hierro $(\mathrm{Fe})$, azufre $(\mathrm{S})$, cobre $(\mathrm{Cu})$, magnesio $(\mathrm{Mg})$, calcio $(\mathrm{Ca})$, cloro $(\mathrm{Cl})$, aluminio ( $\mathrm{Al})$, manganeso $(\mathrm{Mn}), \mathrm{pH}$ y la conductividad eléctrica (CE) que pueden incidir en la regeneración poblacional de la especie, se analizaron muestras de suelo de El Orquidiario y San Bernardo. Dentro de cada localidad se establecieron aleatoriamente seis parcelas de $1 \mathrm{~m}^{2}$ de las que se obtuvo $1 \mathrm{~kg}$ de suelo. Cada muestra de suelo extraído se guardó en una bolsa de plástico y posteriormente se obtuvo en laboratorio el $\mathrm{pH}$ y la conductividad eléctrica a través de un potenciómetro marca HANNA ${ }^{\circledR}$ modelo HI 2211. Además, se analizó la cantidad de 12 elementos en el suelo: aluminio ( $\mathrm{Al})$, amonio $\left(\mathrm{NH}_{4}\right)$, arsénico (As), azufre $(\mathrm{S})$, calcio $(\mathrm{Ca})$, cloro $(\mathrm{Cl})$, cobre $(\mathrm{Cu})$, fósforo $(\mathrm{P})$, hierro $(\mathrm{Fe})$, manganeso $(\mathrm{Mn})$, magnesio $(\mathrm{Mg})$ y nitrato $\left(\mathrm{NO}_{3}{ }^{-}\right.$), usando el aparato de análisis de suelos modelo STH-14 LaMotte.®.

Dado que en cada localidad la especie se distribuye en fragmentos y el bosque contiguo que rodea el fragmento carece de la especie, también se analizaron las características edáficas de esta área sin O. mexicana. Las muestras de suelo en el área sin O. mexicana. fueron colectados entre $20 \mathrm{~m}$ y $50 \mathrm{~m}$ de distancia de donde termina la presencia de la especie. De igual manera, en esta área se tomaron seis muestras de suelo, siguiendo los procedimientos de colecta y análisis antes mencionados. Para la identificación de las variables edáficas más sensibles a la regeneración poblacional de O. mexicana, se utilizó un análisis de componentes principales al evaluar los sitios con y sin presencia de la especie. Antes de aplicar dicho análisis se comprobó, mediante la prueba de esfericidad de Bartlett, si la correlación entre las variables analizadas era lo suficientemente grande para justificar la factorización de la matriz de coeficientes de correlación. Para ambos análisis se utilizó el programa estadístico XLSTAT v.1.0.1 (Addinsoft, 2014). 


\section{Resultados}

\section{Producción de semillas}

Se encontraron diferencias significativas en la producción de semillas entre 2014 y 2015 ( $p<0.05$; $q=8.33$ ) y entre localidades $(\mathrm{p}<0.05 ; \mathrm{q}=12.213)$. La población de San Bernardo tuvo una producción promedio de semillas mayor en $2014\left(38 \mathrm{~m}^{-2} \pm 12 \mathrm{~m}^{-2}\right)$ que en $2015\left(15 \mathrm{~m}^{-2} \pm 6 \mathrm{~m}^{-2}\right)$, patrón similar al obtenido en El Orquidiario $\left(125 \mathrm{~m}^{-2} \pm 32\right.$ $\mathrm{m}^{-2}$ y $10 \mathrm{~m}^{-2} \pm 4 \mathrm{~m}^{-2}$, respectivamente). Se encontró, además, que el peso promedio de las semillas mostró diferencias entre localidades $(p<0.05$; $q=13.13)$, donde las semillas de El Orquidiario mostraron un peso mayor $(0.231 \mathrm{~g} \pm 0.022 \mathrm{~g})$ que en San Bernardo (0.210 g $\pm 0.05 \mathrm{~g})$.

\section{Viabilidad de semillas (rayos X)}

Las radiografías mostraron la presencia de un porcentaje similar de semillas sanas (45.7\%) y de semillas dañadas en el endospermo (41.4\%) y un porcentaje menor de vacías $(12.9 \%)$ (Fig. 2).

\section{Dispersión}

La distancia promedio alcanzada en el experimento de dispersión fue de $13.6 \mathrm{~m}$, con una distancia máxima de 35 m y una mínima de $3 \mathrm{~m}$. No se encontró una relación significativa entre el peso de la semilla y la distancia de dispersión. Sin embargo, se obtuvo una correlación significativa entre el tamaño de la bráctea trilobada y la distancia $\left(\mathrm{p}<0.001 ; \mathrm{r}^{2}=0.845\right)$.

\section{Crecimiento y supervivencia de plántulas}

La supervivencia de las plántulas con y sin exclusión monitoreadas en las localidades se muestra en la figura 3. La depredación de plántulas sin exclusión fue baja en ambas localidades $(<7 \%)$ y fue ausente en condiciones con exclusión. Además, en ninguna de las localidades se registró depredación en las hojas de las plántulas. San Bernardo tuvo mayor supervivencia de plántulas que El Orquidiario $(p<0.001 ; F=25.15)$ en condiciones con y sin exclusión (Fig. 3). En las dos localidades la supervivencia en la condición sin exclusión fue mayor a la condición con exclusión. Al final del análisis, la mortalidad de las plántulas se asoció con la presencia de exclusión al ser la mortalidad en esta condición de 100\% y 80\% para El Orquidiario y San Bernardo, respectivamente (Fig. 3). Al final del estudio, San Bernardo mostró un mayor crecimiento en altura que El Orquidiario $(9.62 \mathrm{~cm} \pm 1.73 \mathrm{~cm}$ y $6.23 \mathrm{~cm} \pm 1.53 \mathrm{~cm}$, respectivamente). En San Bernardo se observó que las plántulas sin exclusión mostraron mayor crecimiento durante los primeros cuatro meses, comportamiento que se invirtió a partir del quinto mes (Fig. 4). Se encontraron diferencias significativas $(\mathrm{p}<0.001 ; \mathrm{F}=15.43$ ) en el crecimiento de las plántulas entre localidades, pero no entre plántulas con y sin exclusión. Al igual que la altura, el diámetro promedio fue significativamente mayor ( $\mathrm{p}<$ 0.001; $\mathrm{F}=24.21)$ en San Bernardo que en el Orquidiario $(0.125 \mathrm{~cm} \pm 0.021 \mathrm{~cm}$ y $0.09 \mathrm{~cm} \pm 0.025 \mathrm{~cm}$, respectivamente). Sin embargo, en San Bernardo, el diámetro de las plántulas con exclusión fue siempre mayor que el de las plántulas sin exclusión (Fig. 5).

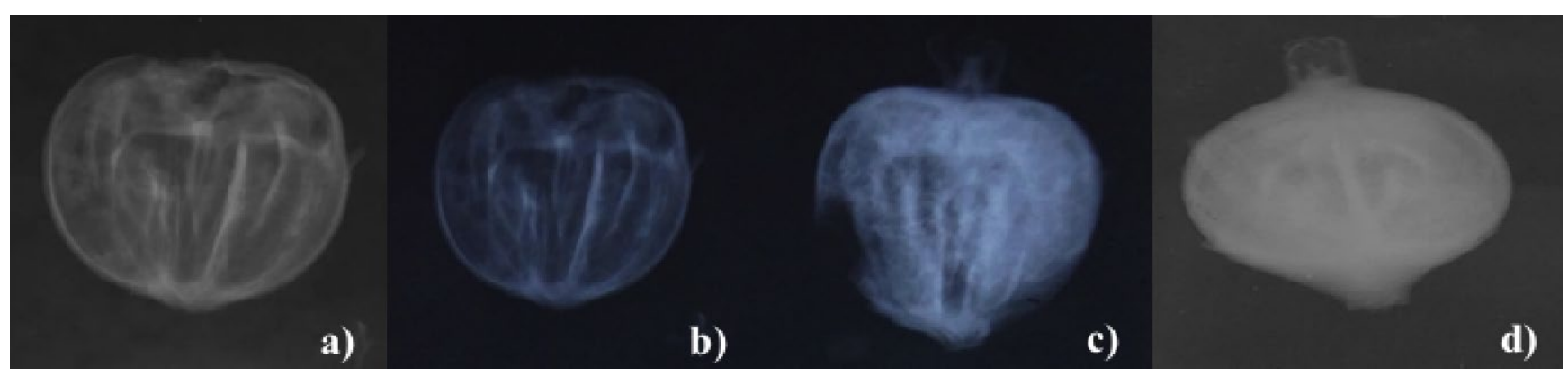

FIGURA 2. Radiografías de semillas de O. mexicana.

a) y b) Semillas vacías; c) Semilla con endospermo dañado; d) Semillas con endospermo en buen estado. 


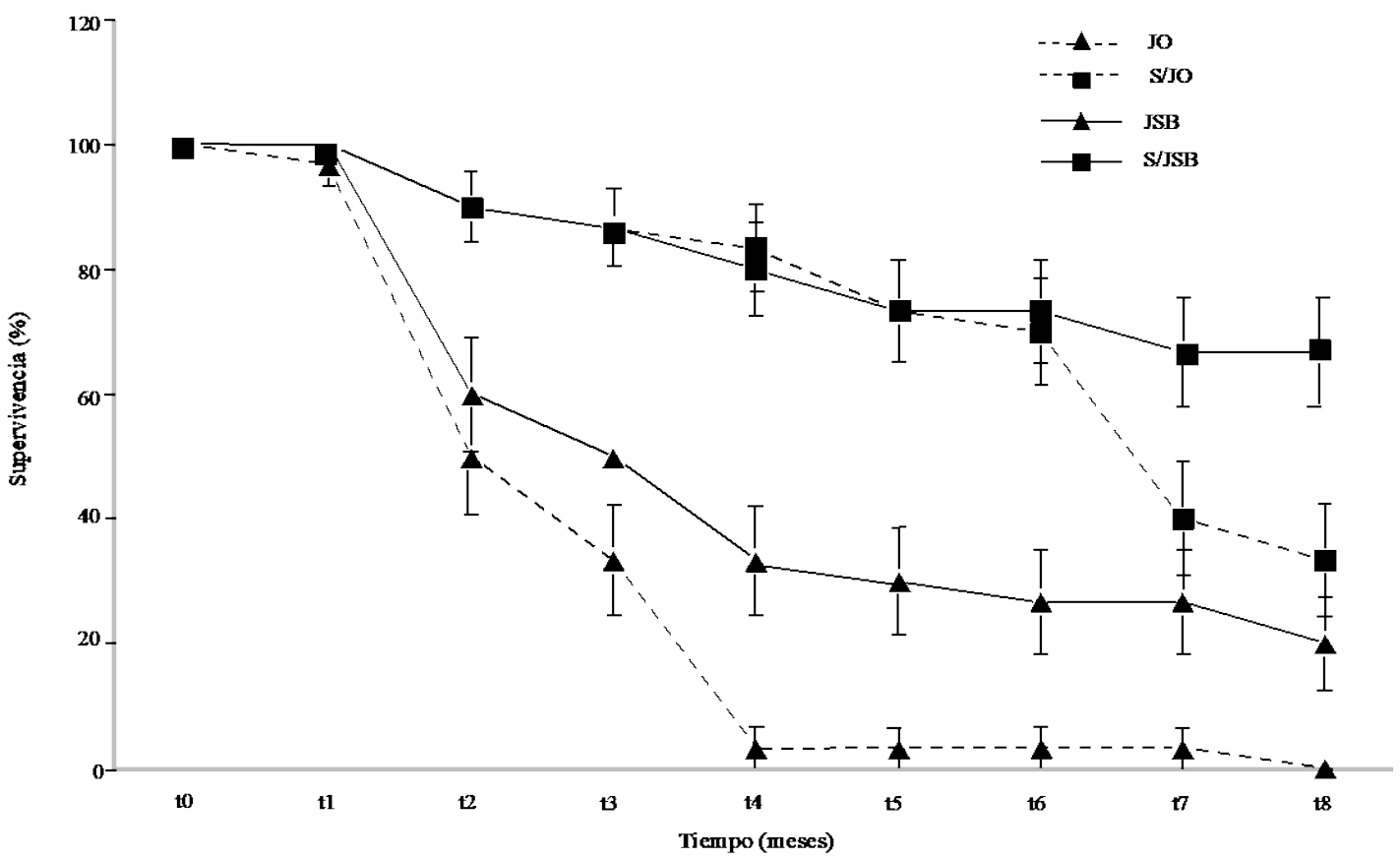

FIGURA 3. Porcentaje de supervivencia de plántulas en San Bernardo y El Orquidiario, con y sin exclusión por ocho meses.

JO: El Orquidiario con exclusión; S/JO El Orquidiario sin exclusión; JSB: San Bernardo con exclusión; S/JSB San Bernardo sin exclusión

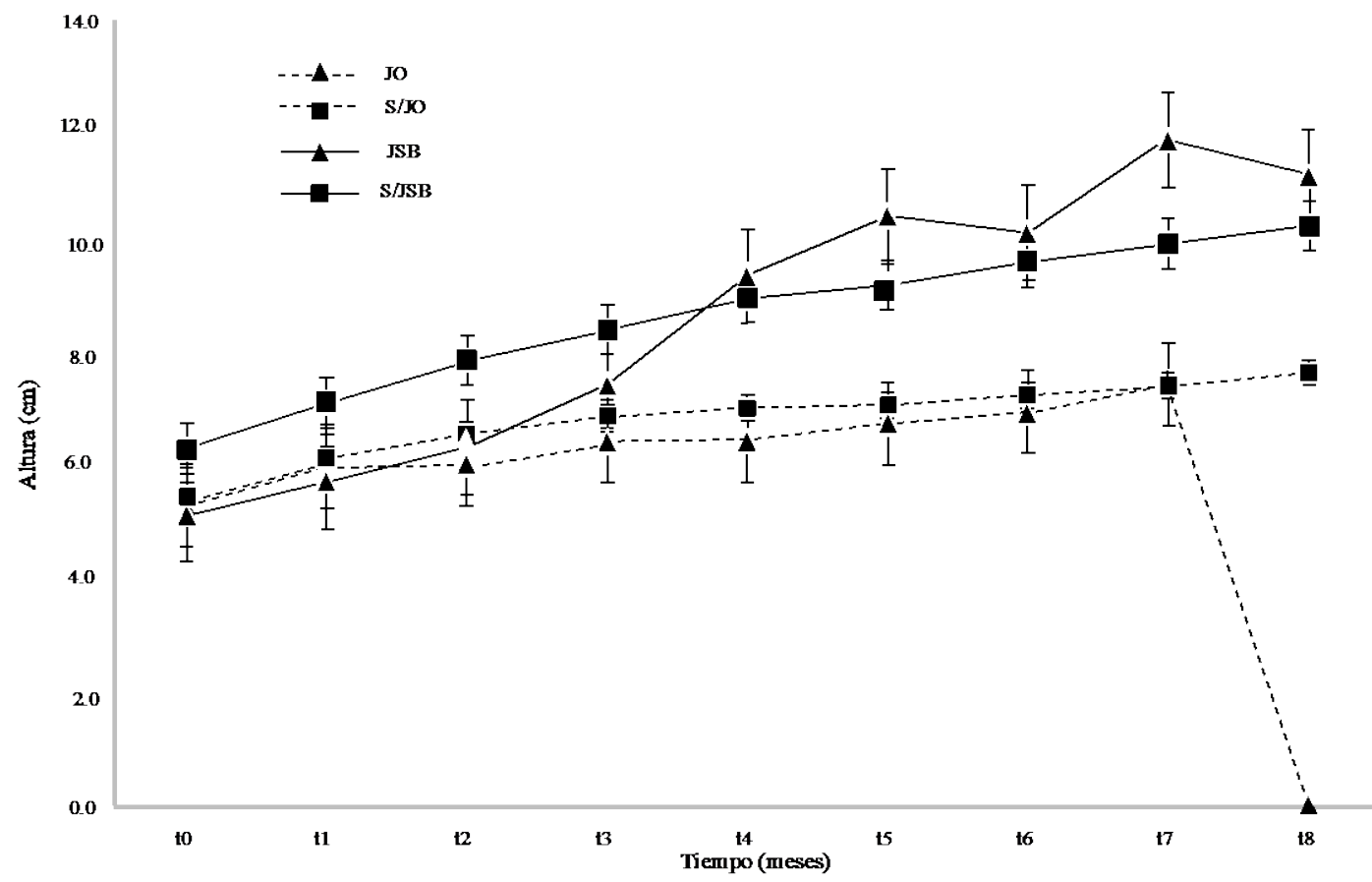

FiguRA 4. Crecimiento de la altura de plántulas en San Bernardo y El Orquidiario, con y sin exclusión por ocho meses.

JO: El Orquidiario con exclusión; S/JO El Orquidiario sin exclusión; JSB: San Bernardo con exclusión; S/JSB San Bernardo sin exclusión 


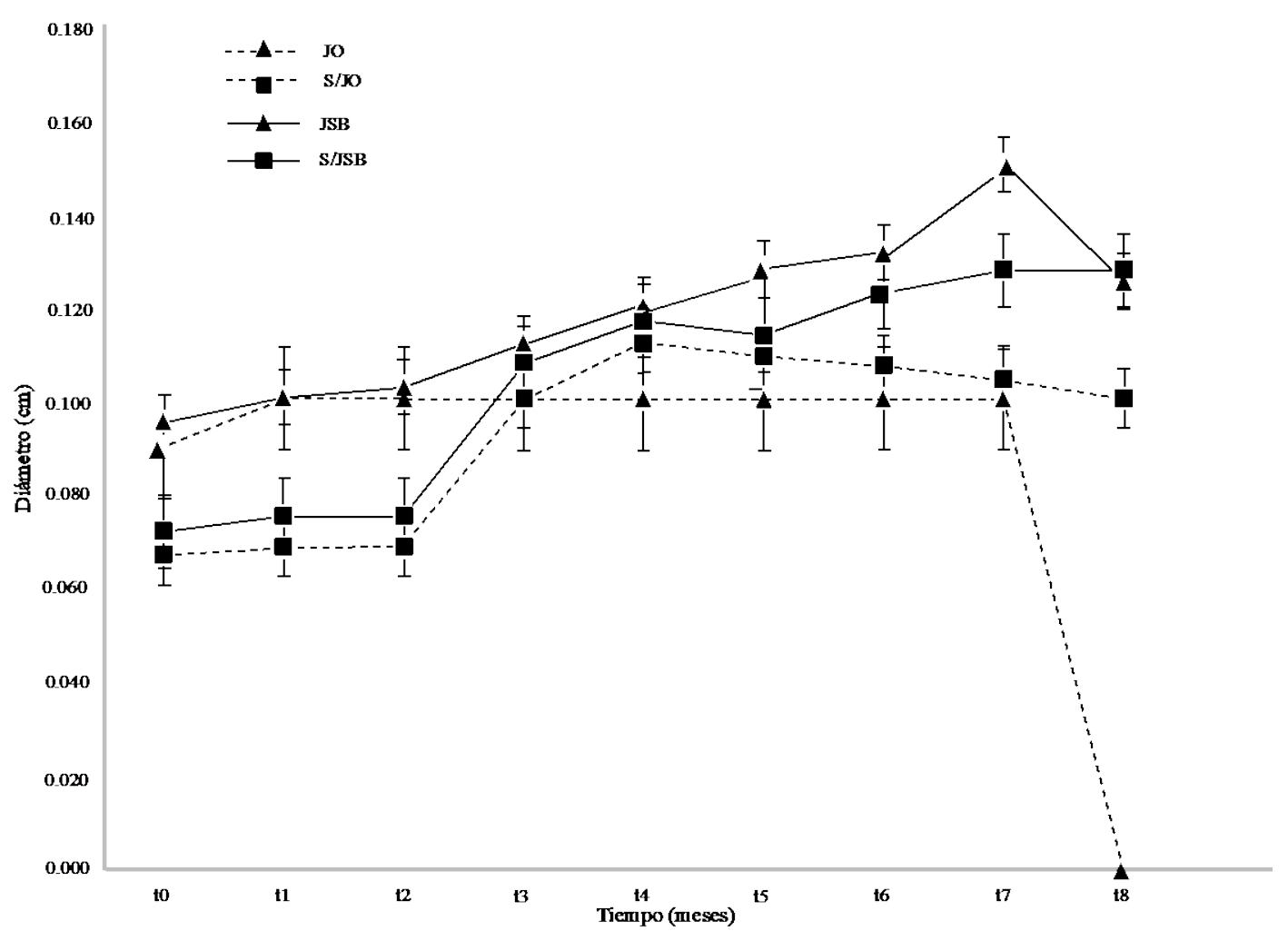

FIGURA 5. Incremento en el diámetro del tallo de las plántulas en San Bernardo y El Orquidiario, con y sin exclusión durante ocho meses.

JO: El Orquidiario con exclusión; S/JO El Orquidiario sin exclusión; JSB: San Bernardo con exclusión; S/JSB San Bernardo sin exclusión.

\section{Estructura del dosel y transmisión de la radiación en el sotobosque}

Los registros en los atributos analizados de la estructura del dosel (apertura del dosel) y de la radiación transmitida (directa, difusa y total) por el dosel del bosque, con excepción del índice de área foliar fueron mayores en la condición de exclusión. Sin embargo, no se encontraron diferencias significativas en los atributos analizados entre localidades (El Orquidiario y San Bernardo), ni entre condiciones de exclusión (con y sin exclusión) (Tabla 1).

\section{Análisis edáficos}

En la figura 6 se presentan los resultados del análisis de componentes principales, donde se observó que existe separación significativa $(\mathrm{p}<0.033)$ de los resultados según el sitio de muestreo (con y sin O. mexicana) dentro de las localidades estudiadas. Los dos primeros componentes con criterio de valor propio (> 1) explicaron $79 \%$ de la variabilidad de los datos, distribuidos en $45.56 \%$ para el primer factor y $33.44 \%$ para el segundo. El primer factor agrupó y separó a los sitios con presencia de O. mexicana por su mayor contenido de calcio y magnesio, mientras que los sitios con ausencia de la especie están dispersos y con un contenido menor de dichos nutrientes y mayor cantidad de otros $(\mathrm{Cu}, \mathrm{P}, \mathrm{Al}, \mathrm{pH})$. El estadístico de Bartlett mostró correlación entre las variables $\left(p<0.001 ; \chi^{2}=114.27\right)$.

\section{DISCUSIÓN}

\section{Producción de semillas}

Este trabajo aporta conocimientos básicos de la regeneración poblacional de $O$. mexicana, en donde se destaca, en primera instancia, una producción de semillas que fluctúa entre años y difiere entre localidades. Esta fluctuación en la producción de semillas se ha observado en otras especies de árboles de vida larga (Álvarez-Aquino y Williams-Linera, 2002; Clark-Tapia et al., 2018), debido a las 
TABLA 1. Atributos de la estructura del dosel y porcentaje de radiación transmitida por el sotobosque en El Orquidiario (Ots) y San Bernardo (SBts), y en las condiciones con exclusión (JO y JSB) y sin exclusión (SJO y SJSB) en las localidades de estudio.

\begin{tabular}{|c|c|c|c|c|c|}
\hline & $\% A D$ & $I A F$ & $\% R D T$ & $\% R F T$ & $\% R T T$ \\
\hline Ots & $24.11 \pm 7.82$ & $1.93 \pm 0.45$ & $14.63 \pm 7.61$ & $15.73 \pm 7.81$ & $17.57 \pm 7.07$ \\
\hline JO & $29.64 \pm 4.86$ & $1.64 \pm 0.35$ & $15.84 \pm 8.17$ & $18.09 \pm 6.83$ & $20.72 \pm 8.47$ \\
\hline SJO & $23.57 \pm 5.91$ & $1.96 \pm 0.37$ & $13.02 \pm 6.12$ & $13.58 \pm 5.98$ & $15.57 \pm 5.04$ \\
\hline SBts & $21.60 \pm 6.77$ & $2.13 \pm 0.45$ & $19.68 \pm 9.15$ & $17.21 \pm 6.83$ & $19.97 \pm 7.44$ \\
\hline JSB & $27.28 \pm 5.96$ & $1.76 \pm 0.39$ & $22.19 \pm 9.89$ & $18.27 \pm 6.67$ & $20.55 \pm 8.89$ \\
\hline SJSB & $20.78 \pm 5.93$ & $2.16 \pm 0.36$ & $17.81 \pm 9.46$ & $15.46 \pm 5.88$ & $18.77 \pm 7.98$ \\
\hline
\end{tabular}

\%AD: Porcentaje de apertura del dosel; IAF; índice de área foliar, \%RDT: porcentaje de radiación directa transmitida; \%RFT: porcentaje de luz difusa transmitida; \%RT: porcentaje de radiación total transmitida

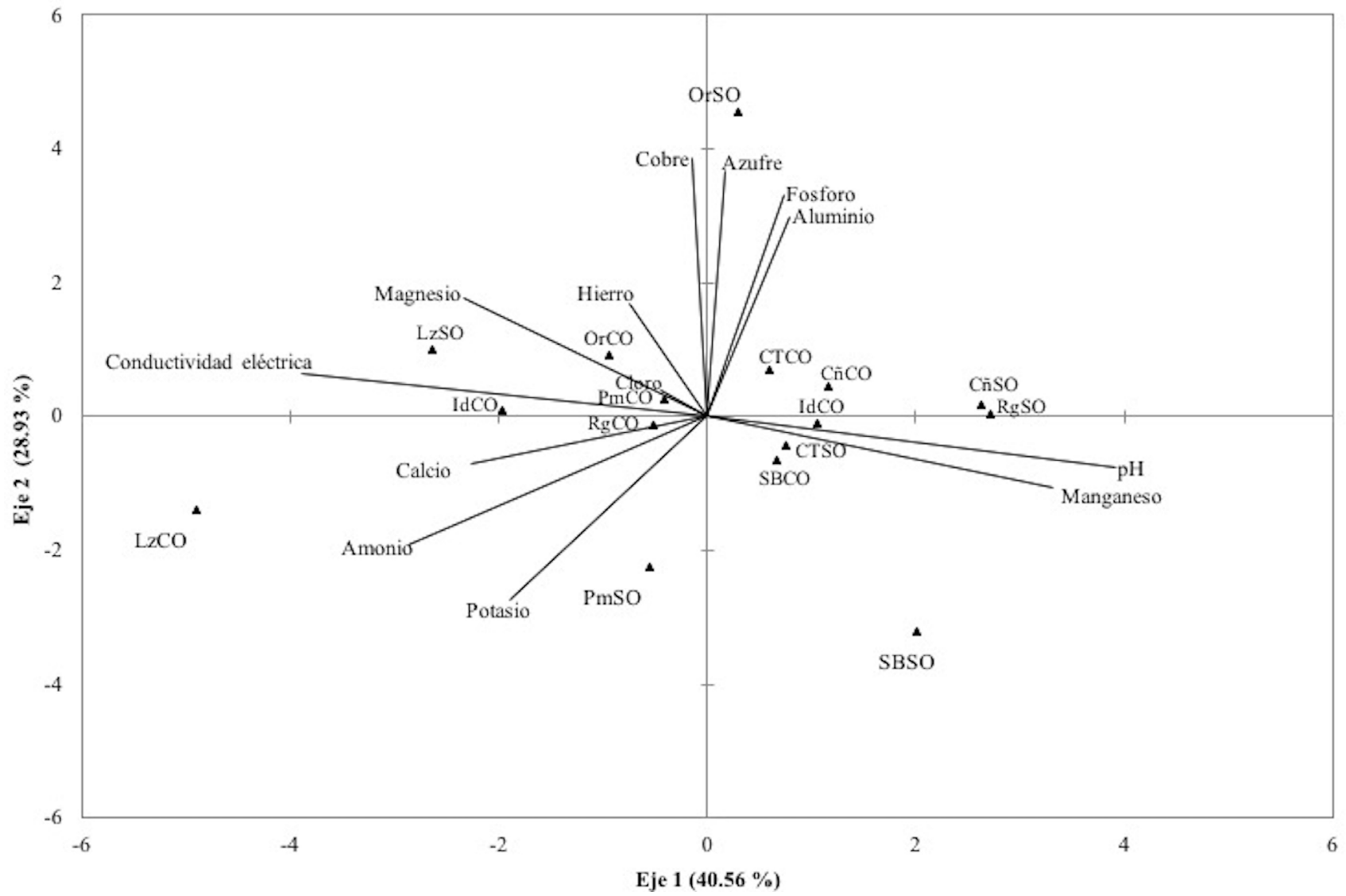

FIGURA 6. Análisis de componentes principales a partir de muestras de suelo tomadas de las localidades de estudio con y sin presencia de O. mexicana (CO y SO, respectivamente).

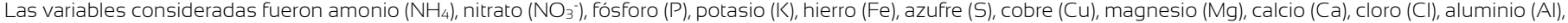
manganeso (Mn), pH y la conductividad eléctrica (CE). 
variaciones climáticas, a la diferencia en historias de vida de las especies y a la presencia de disturbios (Camacho-Cruz y González-Espinosa, 2002; Lehouck et al., 2009).

La producción irregular de semillas es atribuida a factores climáticos, particularmente a la variación en la precipitación y temperatura entre años, factores asociados que causan una producción sincronizada e intermitente de semillas (conocida en inglés como masting) (AlfonsoCorrado et al., 2014; Clark-Tapia et al., 2018). En contraste, la diferencia en la producción de semillas entre localidades se atribuye a la presencia de disturbio en El Orquidiario, dada su cercanía al pueblo de La Esperanza y la similitud con San Bernardo en las condiciones ambientales y orográficas. Aún cuando el efecto del disturbio no fue evaluado, este influye en la calidad y el movimiento de polen con el tipo e intensidad de disturbio, con efectos negativos en la producción de semillas (Camacho-Cruz y González-Espinoza, 2002).

\section{Dispersión}

La dispersión de las semillas en O. mexicana, es predominantemente por viento, la presencia de una bráctea trilobada, no solo es un atributo base para la clasificación taxonómica dentro del género Oreomunnea (Russell y Cohn, 2012), sino una adaptación de la semilla que le permite ser dispersada por viento (anemócora), y a mayor tamaño de la bráctea la distancia de dispersión se incrementa, independientemente del tamaño de la semilla. La distancia de dispersión permite a la semilla alejarse de la madre, ocupar nuevos espacios exentos de fuerte competencia y disminuir la probabilidad de cruzas entre parientes (Rovere y Premoli, 2005; Pascual-Mendoza, 2014).

Aun cuando la distancia promedio de dispersión con viento de baja intensidad fue menor a $14 \mathrm{~m}$, esta se encuentra dentro del intervalo de dispersión obtenido en especies forestales con semillas aladas (Rovere y Premoli, 2005) y podría esperarse que sea mayor en presencia de viento de mayor intensidad. No obstante, la distancia de dispersión de las semillas parece ser suficiente para evitar cruzamiento con grupos familiares de acuerdo con estudios de la estructura y distribución genética espacial que sugieren que las poblaciones de la especie no se encuentran estructuradas genéticamente debido a un flujo génico moderado (Pascual-Mendoza, 2014).

\section{Viabilidad de semillas (rayos $X$ )}

Los insectos parásitos de las semillas son un factor que limita la viabilidad y afectan el establecimiento de una especie (Pacheco-Cruz, 2016). El análisis de viabilidad de semillas presentó un porcentaje alto de parasitismo, similar al observado en especies del orden Fagales $(20 \%$ a $70 \%$ en encinos), (Csóka y Hirka, 2006, Alfonso-Corrado et al., 2014). Este grupo es susceptible a ser parasitado por curculiónidos (Curculionidae) en todo el mundo (Csóka y Hirka, 2006; González-Salvatierra, Badano, Flores y Rodas, 2013, Alfonso-Corrado et al., 2014). Estos insectos comúnmente llamados "picudos" o "gorgojos" (Arnett, Thomas, Skelly y Frank, 2002), son una de las principales causas de pérdida de semillas viables con efectos significativos en la regeneración de las poblaciones (Harper, 1977, Aguilar-Peralta, González-Rodríguez, GonzálezEsquivel y Cuevas-Reyes, 2016). El curculiónido encontrado en $O$. mexicana, es una especie en proceso de descripción, estudios futuros podrían evaluar si la abundancia y el efecto en las semillas de este parásito varía con la producción de semillas entre años (Clark-Tapia et al., 2018).

\section{Crecimiento y supervivencia}

La depredación de plántulas fue menor a 10\%, lo cual es un patrón que contrasta con lo encontrado por AvendañoYánez et al. (2014) quienes señalan que la principal causa de mortalidad en plántulas de O. mexicana es la herbivoría en el bosque de niebla del estado de Veracruz. A pesar de que no se evaluó la causa de mortalidad de las plántulas, se registró que previo a su muerte, la superficie foliar manifestó manchas, decoloración y necrosis. No se descarta una enfermedad provocada por agentes patógenos, requerimientos de macronutrientes o efectos dependientes de la densidad sobre la radiación solar en el sotobosque, 
aspectos que deberán ser explorados en el futuro, así como la búsqueda de depredadores potenciales. No obstante, aunque $O$. mexicana es una especie catalogada como esciófita, sus plántulas son sensibles a la cantidad de luz que absorben (Avendaño-Yánez et al., 2014).

La mayor mortalidad en plántulas se registró en condiciones de exclusión en las que hubo una modificación en la intercepción de la intensidad luminosa que se filtra a través del dosel, debido a la malla de la jaula. Experimentos en campo con plántulas de la especie trasplantadas desde invernadero sugieren que la humedad (Atondo-Bueno et al., 2016) y la apertura del dosel (Avendaño-Yánez et al., 2014; Atondo-Bueno et al., 2016) determinan la calidad y cantidad de radiación que llega al estrato del sotobosque, factores necesarios durante las primeras fases de la vida de $O$. mexicana. Este resultado sugiere que la especie, en su etapa inicial, requiere de condiciones específicas de estas variables ambientales para su establecimiento y supervivencia, similar a lo encontrado en diversas especies del orden Fagales donde la supervivencia de las plántulas es regulada por una multiplicidad de factores climáticos y edáficos (Hölscher, Dunker, Harbusch y Corre, 2009; Pérez-López et al., 2013), factores dependientes de la densidad y de disturbio antrópico (Alfonso-Corrado et al., 2017), así como asociaciones con hongos micorrízicos (Corrales et al., 2016a). Además, la diferencia en el nivel de disturbio entre localidades sugiere que este puede ser un factor importante en regular el crecimiento y la supervivencia de las plántulas de O. mexicana. No obstante, que Avendaño-Yáñez et al. (2016) en Veracruz señalaron resultados contrastantes para el crecimiento y la supervivencia de plántulas debido al disturbio, se sugiere a futuro, analizar el efecto de este proceso para el bosque de niebla de la Sierra Juárez.

\section{Análisis edáficos}

Las características físicas, químicas y biológicas de los suelos son un factor esencial que incide positiva o negativamente en la regeneración de las especies (Cuevas, García, Vázquez y Núñez, 2008; Alvarado-Villafañe, 2011; Corrales et al., 2016a). Para O. mexicana se observa que ciertas características físicas del suelo, como la humedad, favorece la emergencia de plántulas (Atondo-Bueno et al., 2016), además de que el crecimiento de la especie y asociaciones micorrízicas que permiten su desarrollo pueden ser afectadas por elementos químicos del suelo (Corrales et al., 2016a; Corrales, Mangan, Tuner y Dalling, 2016b). En este estudio, se encontraron diferencias en la concentración de elementos químicos del suelo (Ca, $\mathrm{Mg}$, $\mathrm{Cu}, \mathrm{P}, \mathrm{Al}$ y $\mathrm{pH}$ ) entre áreas con presencia y ausencia de la especie, esto sugiere que ciertos macronutrientes son esenciales para su establecimiento y desarrollo. Esto no es exclusivo de la especie, por ejemplo, Cuevas et al. (2008) señalan que la presencia de magnesio soluble favorece al establecimiento de Nectandra rudis C.K. Allen en el bosque de niebla de la Sierra de Manantlán, Jalisco, México. Esta diferencia entre áreas a escala de localidad, indica cierta especificidad edáfica, ya sea en humedad (Atondo et al., 2016), o en nutrientes y asociaciones micorrízicas (Corrales et al., 2016b) aspecto que puede contribuir directamente en el patrón de distribución fragmentado y discontinuo de la especie en la Sierra Juárez.

\section{CONCLUSIONES}

Los resultados de este estudio muestran que Oreomunnea mexicana presenta una regeneración escasa in situ. Entre los factores que contribuyen a la pobre regeneración, son: 1) fluctuación anual en la producción de semillas, 2) alta depredación de semillas por curculiónidos y 3) requerimientos específicos de radiación solar. Asimismo, la supervivencia y crecimiento de las plántulas requieren de condiciones edáficas y de luz solar específicas, lo que favorece su distribución discontinua y monodominancia, sin embargo, la herbivoría no es un factor importante para su establecimiento inicial. Este trabajo es un primer aporte a la regeneración de la especie in situ para el bosque de niebla más conservado de México, sin embargo, dada la baja viabilidad y germinación de la especie, así como los requerimientos de microhábitats específicos para su establecimiento y crecimiento, se recomienda continuar con los estudios de regeneración poblacional; además de estudios que evalúen diversos requerimientos de las 
plántulas de luz y suelo, esto como estrategia de reforestación y conservación de la especie. En el campo, a través de un sistema silvícola que genere diversos tratamientos de iluminación y suelo. En el laboratorio, con cámaras de germinación donde se regulen los niveles de iluminación y variedad de suelo. Finalmente, la producción de plantas micorrizadas en vivero sujetas a distintas condiciones de iluminación y suelo.

\section{RECONOCIMIENTOS}

Los responsables del presente trabajo desean agradecer a las autoridades agrarias de la comunidad de Santiago Comaltepec por la colaboración y ayuda para acceder a las áreas de estudio brindadas. Este trabajo fue financiamiento por el Fondo Fomix (Conacyt-Gobierno del Estado de Oaxaca) con número de proyecto 195425.

\section{REFERENCIAS}

Addinsoft. (1995-2014). XLSTAT. Statistical Software. BroNY, USA: Addinsoft, Inc.

Aguilar-Peralta, J. S., González-Rodríguez, A., González-Esquivel, J. G., \& Cuevas-Reyes, P. (2016). Patrones de depredación de bellotas en especies del género Quercus (Fagaceae) a lo largo de un gradiente altitudinal en el volcán de Tequila, Jalisco. Biológicas, 18(1), 1-9.

Alfonso-Corrado, C., Campos, J. E., Mendoza, A., Aguirre-Hidalgo, V., Valencia Aválos, S., González-Adame, G., Wooden-Garvey, F., \& Clark-Tapia, R. (2014). Restoration-focused germination and development of five central Mexican oak species. Open Journal of Forestry, 4(3), 171-180. doi: 10.4236/ojf.2014.43023.

Alfonso-Corrado, C., Naranjo-Luna, F., Clark-Tapia, R., Campos, J. E., Rojas-Soto, O., Luna-Krauletz, M. D., Bodenhorn, B., GorgonioRamírez, M., \& Pacheco-Cruz, N. (2017). Effects of environmental changes on the occurrences of Oreomunnea mexicana (Juglandaceae) in a biodiversity hotspot cloud forest. Forest, 261(8), 1-15. doi: 10.3390/f8080261

Álvarez-Aquino, C., \& Williams-Linera, G. (2002). Seedling bank dynamics of Fagus grandifolia var. mexicana before and after a mast year in a Mexican cloud forest. Journal of Vegetation Science, 13(2), 179-184. doi: 10.1111/j.1654-1103.2002.tb02037.x/full

Álvarez-Arteaga, G., García-Calderón, N. E., Krasilnikov, P., \& GarcíaOliva, F. (2013). Almacenes de carbono en bosques montanos de niebla de la Sierra Norte de Oaxaca, México. Agrociencia, 47(2), 171-180.
Alvarado-Villafañe, D. S. (2011). Ciclo de nutrimentos en bosques mesófilos de montaña con diferente material parental en la Sierra Triqui-Mixteca, Oaxaca. Tesis de maestría no publicada. Universidad Nacional Autónoma de México. D.F. México.

Anta-Fonseca, S., Galindo-Leal, C., González-Medrano, F., KoleffOsorio, P., Meave del Castillo, J., Moya-Moreno, H., \& VictoriaHernández, A. (2010). Sierra Norte de Oaxaca. En T. Aceves (Ed.). El bosque mesófilo de montaña en México: Amenazas y oportunidades para su conservación y su manejo sostenible (pp. 108-115). Ciudad de México, México: Comisión Nacional para el Conocimiento y Uso de la Biodiversidad.

Arnett, R. H., Thomas, M. C., Skelly, P. E., \& Frank, J. H. (2002). American Beetles Volume II. Polypahaga: Scarabaeoidea through Curculionoidea (1 $1^{\mathrm{a}}$ ed.). Florida, Estados Unidos: Crc Press.

Atondo-Bueno, E. J., López-Barrera, F., Bonilla-Moheno, M., WilliamsLinera, G., \& Ramírez-Marcial, N. (2016). Direct seedling of Oreomunnea mexicana, a threatened tree species from Southeastern, México. New Forest, 47(6), 845-860. doi: 10.007/s11056-016-95482

Avendaño-Yáñez, M. L., Sánchez-Velázquez, R. L., Meave, J. A., \& Pineda-López, M. R. (2014). Is facilitation a promising strategy for cloud forest restoration? Forest Ecology and Management, 329(8), 328-333. doi: 10.1016/j.foreco.2014.01051.

Clark-Tapia, R., Suárez-Mota, M. E., Alfonso-Corrado, C., AguirreHidalgo, V., \& Campos-Contreras J. E. (2016). Clima. En R. Clark-Tapia, M. F. Ramos-Morales, C. Alfonso-Corrado, M. M. Mendoza-Díaz, \& A. Fuente-Carrasco, M. (Eds.). Recursos bídricos de la Sierra Norte de Oaxaca. Caracterización, Diagnóstico y Gestión (pp. 43-47). Oaxaca, México: Universidad de la Sierra Juárez.

Clark-Tapia, R., Mendoza, A., Aguirre-Hidalgo, V., Antúnez, P., Campos, J., Valencia-A., S., Luna-Krauletz, M. D., \& AlfonsoCorrado, C. (2018). Reproducción sexual de Quercus macdongallii, un encino endémico de la Sierra Juárez, Oaxaca. Madera y Bosques, 24(2), e2421617. doi: 10.21829/myb.2018.2421617

Camacho-Cruz, A., \& González Espinosa, M. (2002). Establecimiento temprano de árboles nativos en bosques perturbados de los Altos de Chiapas, México. Ecosistemas, 11(1), 1-9.

Corrales, A., Arnold, E. A., Ferrer, A., Turner, B. L. \& Dalling, J. W. (2016a). Variation in ectomycorrihizal fungal communities associated with Oreomunnea mexicana (Juglandaceae) in a neotropical montane forest. Mycorrbiza, 26(1), 1-17. doi: 10.007/s00572-015-0641-8

Corrales, A., Mangan, S. A., Tuner, B. L., \& Dalling, J. W. (2016b). An ectomycorrhizal nitrogen economy facilitates monodominance in a neotropical forest. Ecological Letter, 19(4), 383-392. doi: $10.1111 /$ ele. 12570 
Csóka, G. \& Hirka, A. (2006). Direct effects of Carpophagous insects on the germination ability and early abscission of oak acorns. Acta Silvatica et Lingnaria Hungarica, 2, 57-68.

Cuevas, G. R., García, M. E., Vázquez, J. A., \& Núñez, N. M. (2008). Estructura poblacional y relaciones ambientales del árbol tropical Nectandra rudis (Lauraceae), una especie rara del occidente de México. Revista de Biología Tropical, 56(1), 247-256. doi. 10.1551.7/RBT.V5611.5521

Frazer, G. W., Canham, C. D., \& Lertzman, K. P. (1999). Gap Light Analyzer (GLA), v 2.0: Imaging software to extract canopy structure and gap light transmission indices from true-color fisheye photographs, user's manual and program documentation. Recuperado de http:// citeseerx.ist.psu.edu/viewdoc/download?.doi=10.1.1.458 .1223

González-Salvatierra, C., Badano, E. I., Flores, J., \& Rodas, J. P. (2013). Germinación, infestación y viabilidad en bellotas de Quercus polimorpha (Schltdl. \& Cham.) tras un año de almacenamiento. Revista Chapingo Serie Ciencias Forestales y del Ambiente, 19(3), 351362. doi: 10.5154/r.rchscfa.2012.07.044

Harper, J. L. (1977). Population biology of plants. (1 a ed.). Inglaterra: Academy Press.

Hölscher, D., Dunker, B., Harbusch, M., \& Corre, M. (2009). Fine root distribution in a lower montane rain forest of Panamá. Biotropica, 41(3), 312-318. doi: 10.1111/j.1744-7429.2009. 00492.x

Lehouck, V., Spanhove, T., Colson, L., Adringa-Davis, A., Cordeiro, N. J., \& Lens, L. (2009). Habitat disturbance reduces seed dispersal of a forest interior tree in a fragmented African cloud forest. Oikos, 118(7), 1023-1034. doi: 10.1111/j.1600-0706.2009.17300

Pacheco-Cruz, N. (2016). Dinámica poblacional y regeneración de Oreomunnea mexicana (Standl.) J.F. Leroy (Juglandaceae), especie relicto del bosque de niebla en la Sierra Juárez, Oaxaca. Tesis de licenciatura no publicada. Universidad de la Sierra Juárez. Oaxaca, México.

Pacheco-Cruz, N., Clark-Tapia, R., Campos-Contreras, J. E., GorgonioRamírez, M., Luna-Krautlez, M. D., Naranjo-Luna, F., \& Alfonso-Corrado, C. (2018). Demografía de Oreomunnea mexicana (Standl.) J. F. Leroy en el bosque de niebla de Sierra Juárez, Oaxaca. Madera y Bosques, 24(2), e2421509. doi: 10.21829/myb.2018.2421509

Palacio-Chávez, R., \& Rzedowski, J. (1993). Estudio palinológico de las flores fósiles del mioceno inferior y mioceno medio de la región de Pichucalco, Chiapas, México. Acta Botánica Mexicana, 24, 1-31.

Parra-Aldana, C. A., Díez-Gómez, M. C., \& Moreno-Hurtado, F. H. (2011). Regeneración natural del roble negro (Colombobalanus excelsa, Fagaceae) en dos poblaciones de la Cordillera Oriental de los Andes, Colombia. Revista Facultad Nacional de Agronomia Medellin, 64(2), 6175-6189.

Pascual-Mendoza, S. (2014). Diversidad y estructura genética de Oreomunnea mexicana (Juglandaceae), especie relicto del bosque de niebla en la Sierra Juárez, Oaxaca. Tesis de licenciatura no publicada. Universidad de la Sierra Juárez. Oaxaca, México.

Pérez-López, P., López-Barrera, F., García-Oliva, F., Cuevas-Reyes, P., \& González-Rodríguez, A. (2013). Procesos de regeneración natural en bosques de encinos: factores facilitadores y limitante. Biológicas, 1(12),18-24.

Primarck, R. B. (2012). A primer of conservation biology (5a ed.). Estados Unidos: Sinauer.

Ponce-Reyes, R., Reynoso-Rosales, V. H., Watson, J. E. M., VanDerWal, J., Fuller, R. A., Pressey, R. L., \& Possingham, H. P. (2012). Vulnerability of cloud forest reserves in Mexico to climate change. Nature Climate Changes, 2(6), 448-452. doi: 10.1038/NCLIMATE1453

Quevedo, A., Schwarzkopf, T., García, C., \& Jerez, M. (2016). Ambiente de luz del sotobosque de una selva nublada andina: estructura del dosel y estacionalidad climática. Revista de Biología Tropical, 64(4), 1-9. doi: $10.15517 /$ rbt.v64i4.21861

Rovere, E. D., \& Premoli, A. C. (2005). Dispersión asimétrica de semillas de Embothrium coccineum (Proteaceae) en el bosque templado de Chiloé, Chile. Ecología Austral, 15, 1-7.

Russell, J., \& Cohn, R. (2012). Oreomunnea mexicana (1ª ed.). Edinburgh, Scotland: Lennex Corp.

Stone, D. E. 1972. New World Juglandaceae. III. A new perspective of the tropical members with winged fruits. Annals of the Missouri Botanical Garden, 59(2), 297-321.

Manuscrito recibido el 24 de agosto de 2018

Aceptado el 3 de abril de 2019

Publicado el 13 de diciembre de 2019

Este documento se debe citar como:

Pacheco-Cruz, N., Clark-Tapia, R., Campos, J. E., GorgonioRamírez, M., Luna-Krauletz, M. D., Naranjo-Luna, F., Corrales, A., \& Alfonso-Corrado, C. (2019). Regeneración de Oreomunnea mexicana (Juglandaceae), una especie relicto, amenazada del bosque de niebla de Sierra Juárez, Oaxaca, México. Madera y Bosques, 25(3), e2531852. doi: 10.21829/myb.2019.2531852

Madera y Bosques por Instituto de Ecología, A.C. se distribuye bajo una Licencia Creative Commons Atribución-NoComercialCompartirlgual 4.0 Internacional. 